University of San Diego

Digital USD

Spring 5-22-2017

\title{
Examining the Neuronal Dopaminergic Pathway Underlying Sleep Behavior and Related Dopamine Sleep Disorders
}

Mary Beth Putz

University of San Diego

Follow this and additional works at: https://digital.sandiego.edu/honors_theses

Part of the Behavioral Neurobiology Commons, Other Genetics and Genomics Commons, and the Other Neuroscience and Neurobiology Commons

\section{Digital USD Citation}

Putz, Mary Beth, "Examining the Neuronal Dopaminergic Pathway Underlying Sleep Behavior and Related Dopamine Sleep Disorders" (2017). Undergraduate Honors Theses. 45.

https://digital.sandiego.edu/honors_theses/45

This Undergraduate Honors Thesis is brought to you for free and open access by the Theses and Dissertations at Digital USD. It has been accepted for inclusion in Undergraduate Honors Theses by an authorized administrator of Digital USD. For more information, please contact digital@sandiego.edu. 
Examining the Neuronal Dopaminergic Pathway Underlying Sleep Behavior and Related Dopamine Sleep Disorders

\author{
A Thesis \\ Presented to \\ The Faculty and the Honors Program \\ Of the University of San Diego
}

By

Mary Beth Putz

Behavioral Neuroscience

2017 


\begin{abstract}
The human brain is an extremely complex organ with approximately 100 billion different neurons that are constantly sending and receiving messages. These messages are sent using the chemical messengers of the brain: neurotransmitters and neuromodulators. Mechanisms of neural control of sleep are substantially conserved across species. Evidence from multiple animal models including flies, zebrafish, and mice shows that the arousal, or wake phase, is regulated by conserved neuromodulators such as dopamine, norepinephrine, and serotonin. Since these neurotransmitter systems are distributed throughout the brain and sub-serve many functions in addition to sleep, the precise circuit mechanisms by which these neurotransmitters regulate sleep remain unknown in any organism. Because of their genetic tractability and mechanistic similarity of sleep to humans, we used Drosophila melanogaster (fruit fly) to study sleep behavior. Our data show that dopamine neurons are critical in controlling sleep behavior and that modification of neurons expressing the dopamine receptor leads to sleep deficits. In addition to analyzing these experimental data, we will also examine the current research regarding established sleep disorders that have a strong dopamine component.
\end{abstract}




\section{Acknowledgements}

Thanks to Dr. Sitaraman, who has been both my academic and research advisor, for her guidance and support throughout this entire process, and without whom this thesis would not be possible.

A huge thanks to my lab partner Tori Coleman, as well as my fellow lab students, Eleanor Bauer, Bridget Fitzgerald, and Margaret Driscoll for their continuous assistance, company, and support in and out of the lab.

Thanks so much to the Honors Program for a great experience over the four years and especially to Dr. Gump and Erin Prickett for their leadership and dedication.

Finally, I would like to thank to my parents and my friends who have been instrumental in supporting, guiding, and encouraging me to pursue my dreams and become the best person I can be. 


\section{Introduction}

Sleep is an essential behavior that can affect one's cognitive processes, immune functioning, memory, and mood, and yet there are still much unknown about how the brain controls and regulates sleep behavior. Even though the field of neuroscience has recently begun to delve into the underlying neural control of sleep, there are many different components of sleep behavior that could still be explored. Within the fields of neuroscience, psychology, and medicine, there is an urgency to study sleep mechanisms for the benefit of all those who may experience sleep disturbances.

However, studying sleep, especially the neuroscience of sleep, is a difficult task due to the complexity of the human brain and the expected ethical restrictions of investigative research. Therefore, it is of utmost importance to identify an animal model where their brains and behaviors are easier to analyze and manipulate. Thus, Drosophila melanogaster, the fruit fly, was used to study sleep/wake behavior. The fruit fly is an excellent model because not only can their sleeping behavior be easily recognized and quantitatively measured, but we can also directly target transgenes into their genomes to analyze any differences in behavior. The fly brain is astonishingly similar to the human brain, especially with controlling sleep, which means that the more we understand the fly brain, the more we can learn about the human brain (Tomita 2017).

When discussing the control of sleep in the brain, one component of this control is the type of neurotransmitter used by the sleep circuits. Present in both Drosophila and humans is the neurotransmitter dopamine. Although dopamine plays many roles in the brain, dopaminergic control of sleep/wake behavior appears to be one of the main forms of regulation of sleep (Potdar 
2013). However, there is little known about the specifics of this control, such as which exact neurons or neural circuits are controlling sleep, and the precise role of dopamine in this system.

Thus, we were able to utilize some of the preexisting genetic tools that target specific neuron clusters within the fly brain that are believed to be implicated in the dopaminergic control of sleep. By targeting these clusters, we aimed to manipulate them in a way that will allow us to potentially learn which of the clusters are in fact controlling sleep, and the mechanisms of that control.

In our preliminary data, there appears to be a significant difference in sleeping behavior in the flies expressing the 54B neuron cluster, which suggests that that cluster of dopamine neurons is associated with regulating sleep behavior.

A final component of this thesis is a literature review analysis of three different sleep disorders that are known to have a dopamine component: Parkinson's Disease, Schizophrenia, and Restless Leg Syndrome. This was included in order to provide some context for the research as the laboratory work could someday apply to the understanding and treatment of the sleep disorders. 


\section{Review of Previous Literature}

There is a growing interest in neuroscience which has led to an increase in research and knowledge about the brain and its many structures and complex mechanisms. How the brain controls sleep is a topic that captures the interest of many as it has important implications to daily functions of all humans. However, due to the complexity of the human brain and the neural mechanisms that control sleep, it is important to first begin in a model organism that closely resembles the human brain, but is simpler and easier to understand.

One such organism often used to study sleep behavior is Drosophila melanogaster, or the fruit fly. Despite the obvious differences between a human and fly, their brains are remarably similar to ours, and there are many structures and mechanisms that are analogous to the human model. Therefore, it is important to have a base knowledge of flies and their brains before moving into the human model.

The fly and human brains both use the neurotransmitter dopamine to communicate between neurons, and both use dopamine in the control of sleep/wake patterns. Dopamine is known to be associated with wake behavior and artificially increasing dopamine generally causes a decrease in sleep behavior (Wisor 2001). Though the mechanisms are not completely understood for either, it is believed that the mechanisms of how dopamine works is very similar in both brains.

Structurally, there are some important similarities and differences that are applicable when studying the mechanisms of sleep. In the fly brain, there are approximately 200 dopaminergic neurons that have been grouped into different clusters. All of these clusters project to the mushroom body (MB), which is a structure in the fly's brain, as well as many other insects, that is known to be involved in olfaction and olfactory memory, the processing of visual 
and mechanosensory input, general higher order processing, as well as the control of sleep behavior (Heisenberg 1998.). The MB extends through the midbrain and is composed of long, densely packed nerve fiber of the Kenyon cells (Heisenberg 1998). Kenyon cells (KCs) are the intrinsic neurons of the mushroom bodies, and the fruit fly is known to have about 2,500. There are three main types of Kenyon cells, as determined by their structural and biochemical differences. There are Kenyon cells that make up the $\gamma$ system, as well as ones that make up the $\alpha \beta$ and $\alpha^{\prime} \beta$ ' systems (Koenig 2016). There is a lot of data regarding the projections into the mushroom body, which include the antennal lobe, another region of the fly brain. However, not as much is known about the efferent pathways that project out of the mushroom body because of the complexity of the system. There is still much research that can be completed in order to explore the output neurons to learn more about how the mushroom body is controlling behaviors in the fruit fly.

In an experiment by Sitaraman et al., they were able to identify specific mushroom body Kenyon cells that are implicated in regulating sleep behavior (2015). It was found that they are connected to specific mushroom body output neurons that then go to specific target regions. They looked directly at dopaminergic neurons innervating the mushroom body in order to study the effects of dopamine on sleep behavior. They identified different dopamine neuron clusters that are potentially involved in the dopaminergic control of sleep, and some of these clusters are the ones used in this research.

Because of the inclusion of human sleep disorders in this research, it is important to understand how sleep is controlled not only in the fly brain, but also in the human brain. The general understanding about dopamine's influence in keeping an organism awake had led to many experiments testing dopamine's effects, with regards to which parts of the human brain 
dopamine has the most influence. One such brain area for dopamine neurons is the ventral tegmental area, or VTA. The ventral tegmental area is located in the midbrain and is close to the substantia nigra. Is it part of two of the most important dopamine pathways: the mesolimbic pathway, which connects the VTA to the nucleus accumbens, and the mesocortical pathway, which connects the VTA to the cortical regions in the frontal lobes. The ventral tegmental area is considered to be associated with the reward system, as well as sleep, and this connection can be seen through the experiments carried out by Lecea et al. The research group identified specific dopamine neurons in the ventral tegmental area. Using a variety of methods, including monitoring calcium levels using fiber photometry in freely behaving mice, they found alterations in VTA dopamine neurons depending on their state of arousal (Lecea et al. 2016). More specifically, they found that when the mice awoke, dopamine neurons in the ventral tegmental area were active and then stayed active for the duration of the time they were awake. Conversely, when the mice were asleep, these neurons were not very active and decreased in activity the longer the mice were asleep. Using chemogenetic and ontogenetic tools, the researchers were able to activate these dopamine neurons, which resulted in the mice staying awake for long periods of time, inconsistent with the behavior of the control group whose VTA dopamine neurons were not being constantly activated. The opposite was also seen: when the dopamine neurons were inactivated, the mice stayed asleep, even when they would normally be active. Taken together, these results show that the dopamine neurons in the VTA, when active, cause wakeful behavior, and when inactive, result in sleep behavior. These results show that not only do the dopamine neurons in the ventral tegmental area specifically not only play an important role in sleep-wake regulation, but also that there is also a potential connection between sleep and a reward pathway. 
Another important brain region regulating sleep containing dopamine neurons is the substantia nigra pars compacta $(\mathrm{SNpc})$. Located in the midbrain, the dopamine neurons in the SNpc has been shown to play an important role in the regulation of sleep. In an experiment by Lima and Andersen, they showed that a 50\% lesion in the substantia nigra pars compacta of rats, and thus a reduction in dopamine neurons, resulted in disruptions in the sleep-wake patterns of the rats. Throughout the five days of recording in both light and dark periods they saw a substantial decrease in the latency to the onset of slow wave sleep (SWS), or in other words, they saw that the onset of slow wave sleep more quickly in lesioned rats than in the control rats. This is relatively consistent with the findings from the Lecea paper, as this also shows that a lack of active dopamine neurons results in more sleep for the animal. A second important finding from this experiment was that the reduction of dopamine neurons in the SNpc resulted in a decrease in the percentage of REM sleep, with a statistically significant correlation between the number of dopamine neurons lost and the percentage decrease of REM sleep on the first day. Thus, these results taken together show that dopamine neurons in the substantia nigra pars compacta play a significant role in the regulation of sleep, especially in regards to REM sleep.

Thus, the significance of the ventral tegmental area and the substantia nigra pars compacta in the regulation of sleep and waking have been shown, but it is also important to recognize their efferent and afferent connections with the dorsal raphe nucleus (DRN), pedunculopontine and laterodorsal tegmental nuclei (PPT/LDT), locus corruleus (LC), lateral hypothalamus (LH), and the basal forebrain (BFB) (Pasquier et al. 2010). For example, Pasquier et al. shows a direct projection from the DRN to the SNpc of the rat, Sakai et al. characterized a direct projection from the SNpc back to the DRN, and a projection from the VTA to the DRN was confirmed by Decavel et al. 


\section{Methods}

\section{Cell-Specific Targeting Technique}

Dopaminergic system. The neuromodulator dopamine is a chemical messenger in the brain. Dopamine neurons, which are neurons that communicate using dopamine, control many behaviors and functions of the body in humans and flies, such as sleep. Biochemically, dopamine is synthesized mainly in the medulla of the adrenal glands, and its direct precursor, L-DOPA, is synthesized from the amino acid tyrosine. The enzyme tyrosine hydroxylase (TH) is responsible for this conversion from tyrosine into L-DOPA, and L-DOPA is converted to converted to dopamine using the enzyme aromatic L-amino acid decarboxylase. Because dopamine is unable to cross the blood-brain barrier, it must be synthesized in the brain. Once dopamine is made, it is transported from the cytosol into synaptic vesicles, where it is stored in these vesicles until it is needed for release by the neurons as a result of an action potential. In the process of activation of neurons, action potentials move from neuron to neuron such that they activate the release of neurotransmitters, such as dopamine, into the synaptic cleft. While in the cleft, dopamine binds to receptors on the post-synaptic neuron, which passes along the message. Neurons are activated by an influx of positive ions via voltage-gated ion channels, which is called depolarization. Neurotransmitters and neuromodulators are taken back up into the pre-synaptic terminal by transporters, which allows the entire process to be continuously repeated. The processes of depolarization and neurotransmitter receptors are particularly relevant for this research, as both systems will be artificially manipulated. 

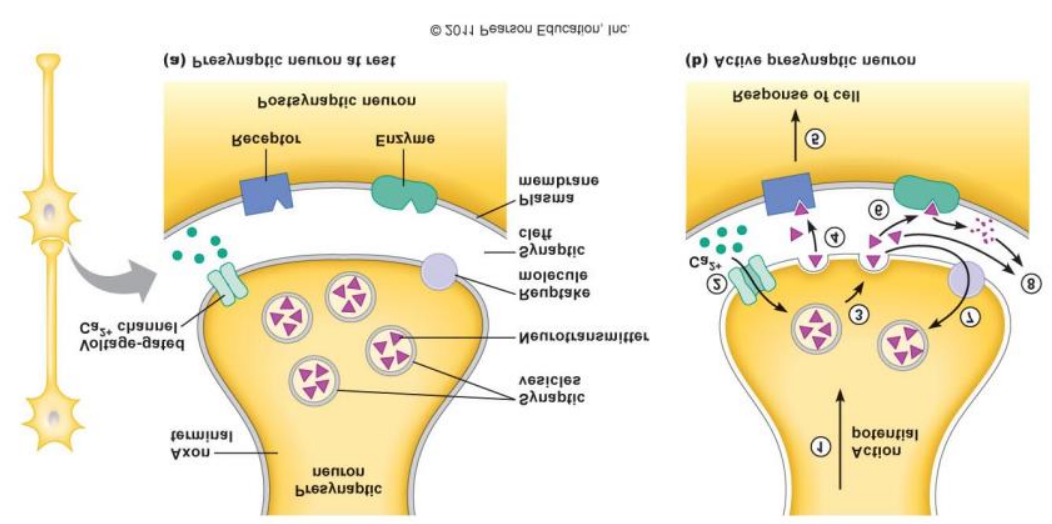

Figure 1. Neurotransmitter release from pre-synaptic neuron. The process of an action potential traveling down the neuron and subsequent neurotransmitter release, binding to receptors, and reuptake

UAS/Gal4 system. The UAS/Gal4 binary system is a transgenic tool for expressing transgenes in specific cells. The ultimate goal is to insert a piece of DNA so that new instructions can be integrated into the genome of the fly, so that it can be expressed in certain cells in the fly's brain. Just like not all genes are expressed in every cell in the body, our goal was to get activation of the transgene to be controlling both spatially and temporally. This system has two pain components: the first is the Gal4 gene and the second is UAS, or upstream activation sequence. Gal4 is a gene obtained from yeast that codes for a transcription factor protein, also called Gal4. As transcription factors do, it binds to an enhancer, which allows expression of genes downstream of the enhancer. In this binary system, UAS is the enhancer to which Gal4 binds. Thus, whenever both Gal4 and UAS are present, there will be activation of the gene downstream of UAS. In order to obtain spatial specificity of expression of the transgene, a tissue specific promoter is attached upstream of Gal4 so that this system is only occurring in the tissues where the promoter is active. Thus, we can directly control expression of where we want Gal4 to bind to UAS and thus where we want expression of the transgene.

Overall, this is a way to artificially control gene expression because Gal4 is not normally present in the DNA of the fly. Its presence does not interfere with other processes of the brain, so 
it is a fairly accurate method of controlled manipulation. Because of the high cost and difficulty in developing a fly that expresses all of these artificial genetic elements, it is more feasible to obtain flies that express one of these genetic differences, and then cross them so that the progeny are expressing all of the genes of interest. Because this research focuses on dopamine, the tissues specified by the promoters were distinct clusters of dopamine neurons that have previously been implicated in controlling sleep behavior.

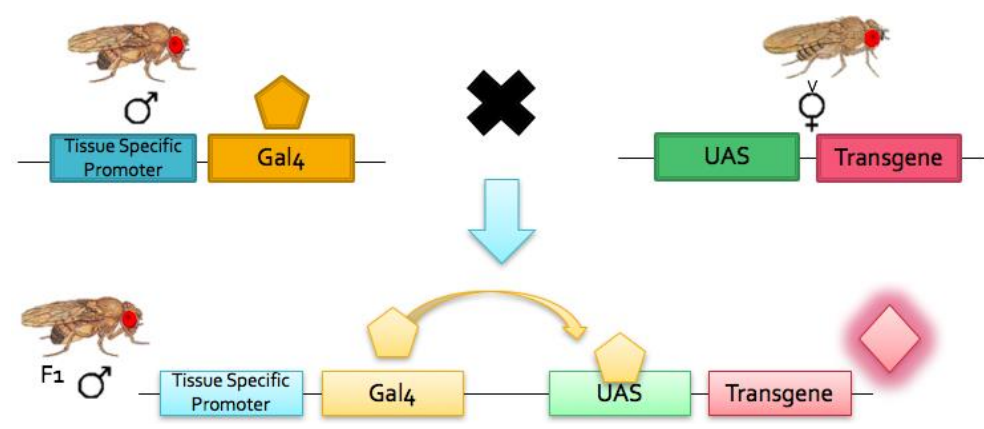

Figure 2. UAS/Gal4 binary system. A visual representation of the binary system and how it drives specific gene expression

Thermal signaling of dopaminergic clusters with dTrpA1. In this research, the specific transgene that was inserted into the genome downstream of UAS was a gene that codes for a chemosensitive cation channel referred to as dTrpA1 or dTrp. This is a channel that allows positively charged ions to pass through, leading to depolarization, which in turn activates a neuron and stimulates neurotransmitter release. Thus, instructions are being added to the genome that could affect the release of dopamine in the brain. However, simply inserting the gene that codes for this channel does not mean that it will immediately be expressed. First, there is still the control in place with using the tissue specific promoter upstream of Gal4, which will specify activity of dTrp to the desired neuron clusters. However, this is not the only form of control, as it is a chemosensitive channel, thus this channel is also only active when the temperature is raised 
above its thermal neutral zone, or raised above $27^{\circ} \mathrm{C}$. Thus, in order to get expression of this channel, not only do Gal4 and UAS need to be present, but the temperature also needs to be raised. If the temperature is below $23^{\circ} \mathrm{C}$, then there should be no activation of the channel and normal neural activity should be observed. Taken in conjunction with the tissue specific promoter, with this temperature-controlled cation channel, we are able to achieve both spatial and temporal specificity of the transgene.

Dopamine receptor knock-out using DopR2 null. The final genetic component of this research is also one of the most important. This is the introduction of the gene for a dopamine receptor, R2, that is nonfunctional. A nonfunctional receptor means that even if dopamine is released from the pre-synaptic neuron, the post-synaptic neuron will not have a way to receive the message. This receptor null background was incorporated into the genome of the flies downstream of both UAS and dTrpA1 so that it could be incorporated into the binary control system of UAS/Gal4. Thus, whenever dTrpA1 is activated and increases release of dopamine in specific dopamine neuron clusters, there will be a non-functional receptor that will potentially mitigate the effects of the activation of dTrp. Previous studies have shown that when flies have dTrp, but no DopR2, and are exposed to heat, they sleep less. Thus, this research will look at the potential of isolating the most important neurons that control sleep, as they will be likely be affected by the addition of a receptor null background.

\section{Mating for Transgenic Progeny}

Fly stocks. Drosophila melanogaster stocks were ordered from HHMI Visiting Scientist program at Yale that included UAS-dTrpA1-DopR2 null, pBD-Gal4, 54B-Gal4, 196B-Gal4, and 328B-Gal4, among others. These flies possessed the necessary genetic elements to utilize the binary control system of UAS/Gal4. 
Genetic Cross. There is one important genetic cross the needs to be performed so that all of the genetic elements will be expressed in the flies that participate in the sleep assays. In order to complete the cross, virgin females, easily recognized by the presence of meconium, were collected from the UAS-dTrpA1-DopR2 null stocks, and were crossed with males from each respective Gal4 stock. They were crossed in vials and then had approximately 4-5 days to mate and for the females to lay eggs until the parent generation was flipped into new vials. This was done for two important reasons. The first was to ensure that once the larvae emerged from their pupa cases, there would be no confusion about which flies were of the F1 generation, and which were the parents. The second was to ensure that we would have a sufficient number of male offspring to use in the sleep assays. This F1 generation expresses not only the Gal4 downstream of a tissue specific promoter, but also UAS upstream of the transgene dTrpA1 and DopR2 null. This cross, from the first day the two genetic categories of flies are introduced until the development of an adult F1 fly is about 12 days, at room temperature. Collection of virgin females for the original cross needs to occur within 8 hours of the female fruit fly eclosing from her pupa case. During these first 8 hours, flies do not mate, and we can be confident in this distinction if a meconium is seen on her abdomen. It is important to only use virgin females so that we can be sure that any eggs she lays contain the vital genetic carried by the Gal4 males.

\section{Sleep Monitor Assay}

Preparing sleep tubes. In order to carry out a sleep experiment, there are a few important components that need to be prepared. The first are the sleep tubes that serve as the home of the fly while its sleep is monitored. A sleep tube is small glass cylinder with openings on both ends, where one end is filled with food and then capped with wax to ensure the food 
does not slip out. The food is a mixture of sucrose and agarose and provides the necessary nutrients for the flies while they are in the sleep tubes.

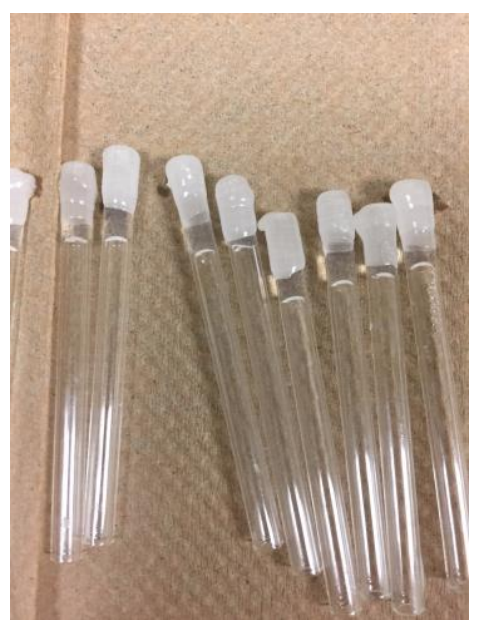

Figure 3. Sleep tubes. An image of the tubes with food and a wax cap

Loading sleep tubes and sleep monitors. The next step is load an individual fly into each tube, and then to temporarily seal the open end using a pipe-cleaner tip as this is the perfect width and allows oxygen to pass through into the sleep tube. Once a tube has one fly and is capped, it is placed into the sleep monitor openings. There are 32 slots, and thus a full sleep monitor has 32 individual flies, 1 in each tube. A rubber band is used to hold the tubes in place so that they do not move around during transport or while in the incubator, as this could potentially introduce error into the data. Each monitor is labeled according to the genetic cross of the flies. 


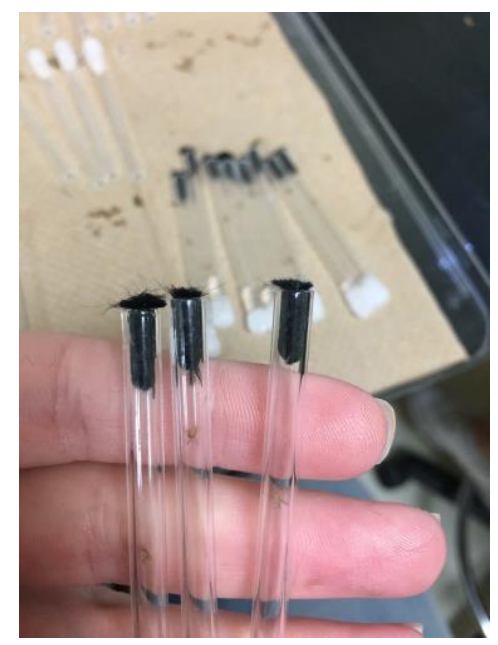

Figure 4. Loading sleep tubes.

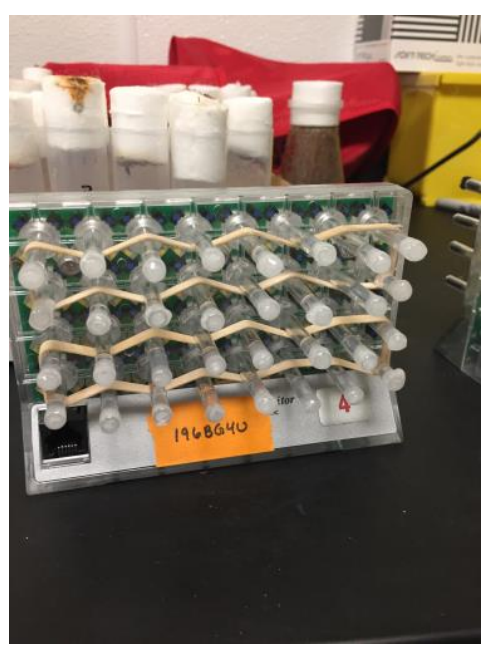

Figure 5. Loading sleep monitors

Loading monitors into incubator. Once the monitors are full, they are transferred into the designated sleep incubators that are equipped to carefully regulate the temperature as well as register the sleep/wake behavior of the flies. Each monitor is plugged into the data compilation device, Drosophila Activity Monitor Protocol, which registers the fly's movements.

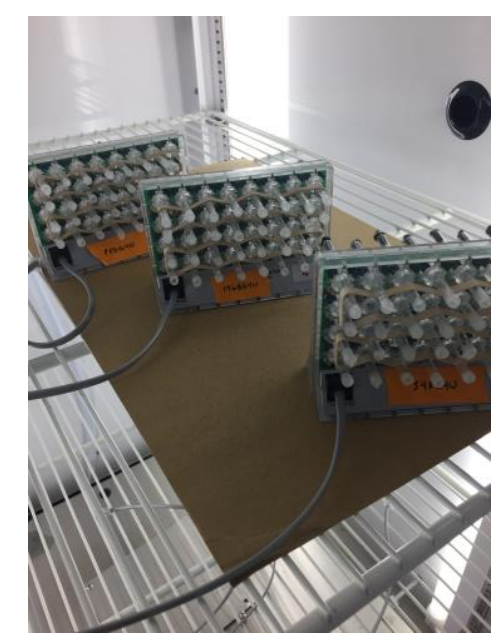

Figure 6. Loading sleep incubators

\section{Experimental Procedures}

Temperature Control. The main experimental manipulation in the research was the adjustment of temperature during the sleep experiment. For the first three days, the temperature 
in the sleep incubators is kept at a constant temperature of $21^{\circ} \mathrm{C}$, which is a standard temperature for the fruit fly. This low temperature means that no activation of $\mathrm{dTrpA} 1$ is occurring. These first three act as a control and a time to establish baseline levels of sleep and waking behaviors in each of the flies. On the fourth day, the temperature is raised to $29^{\circ} \mathrm{C}$, which makes this activation day. This is when the chemosensitive cation channel should be activated in the flies in each of their respective dopamine clusters. On the last day, day five, the temperature is brought back down to $21^{\circ} \mathrm{C}$ as a post-activation control measurement.

\section{Results}

In the first part of the experiment, the ten different genotypes of flies are exposed to an environment of $21^{\circ} \mathrm{C}$ for 3 days in order to establish baseline levels of sleep and waking behavior. Figure 7 shows the genotypes and their relatives amount of total sleep time while at $21^{\circ} \mathrm{C}$.

\section{Degrees}

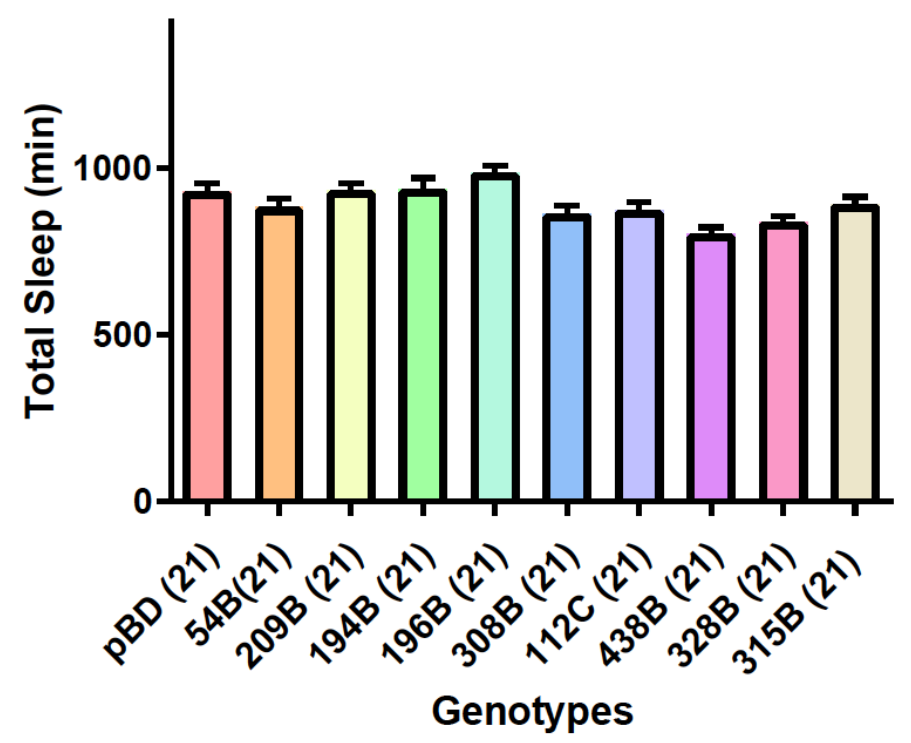

Figure 7. Total sleep time during pre-activation days. Comparing the 10 different Gal4 lines and the total amount of sleep time during days 1-3, before activation of dTrpA1 
The genotype $\mathrm{pBD}$ is the control, which means it does not have any artificial genetic components, and thus should give us a good control with which to compare to the other genotypes. Figure 7 shows that across all of the genotypes, there is little variation in total sleep time. The error bars are small, and none were found to be statistically different from pBD nor each other.

The second part of the experiment was day 4 when the temperature was raised to $29^{\circ} \mathrm{C}$ in order to induce activation of $\mathrm{dTrpA} 1$, the chemosensitive ion channel that increases dopamine release in the specific dopamine cluster denoted by its genotype name.

\section{Degrees}

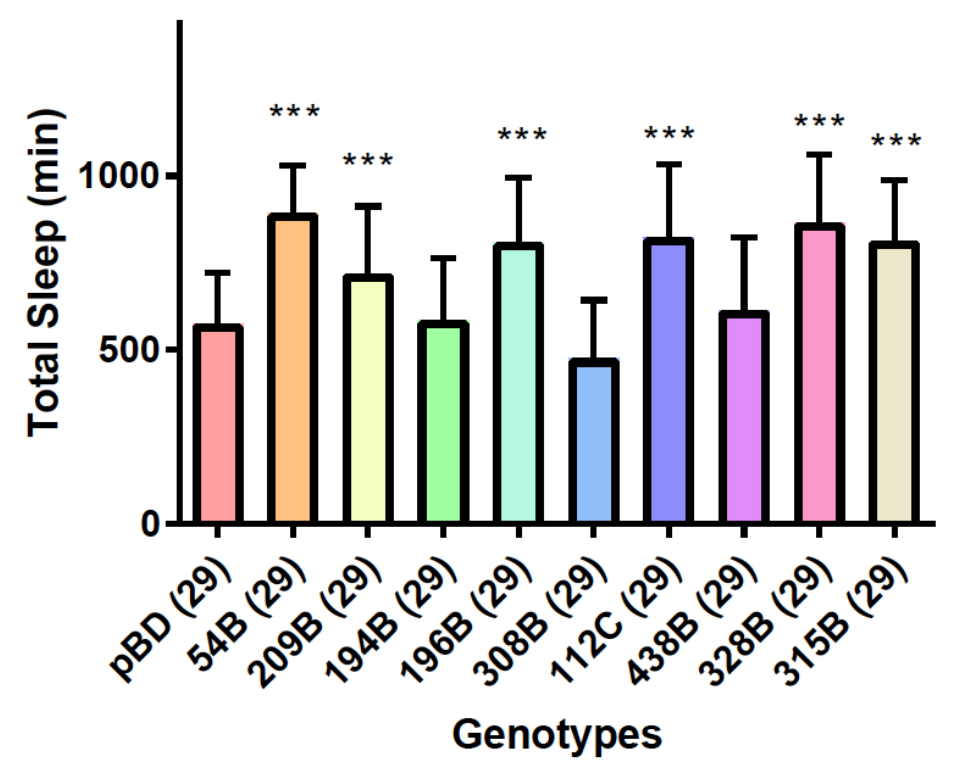

Figure 8. Total sleep time during activation. Comparing the 10 different Gal4 lines and the total amount of sleep time during day 4 and activation of $\mathrm{dTrpA} 1 ; * * *$ denotes statistical significance

Figure 8 shows the same ten genotypes from Figure 7, but with data from activation day. The graph also shows total sleep time, and the measurements of sleep can be compared to both their respective values at $21^{\circ} \mathrm{C}$, as well as the control and to each other. As indicated by the triple 
asterisk, after a one-way ANOVA followed by post-hoc correction, it was found that six separate genotypes had total sleep time that was statistically significant, when compared to both the control, pBD and all genotypes at $29^{\circ} \mathrm{C}$. Figure 9 shows the confidence interval of the one ANOVA comparing each genotype to the control.

\section{5\% Confidence Intervals (Dunnet)}

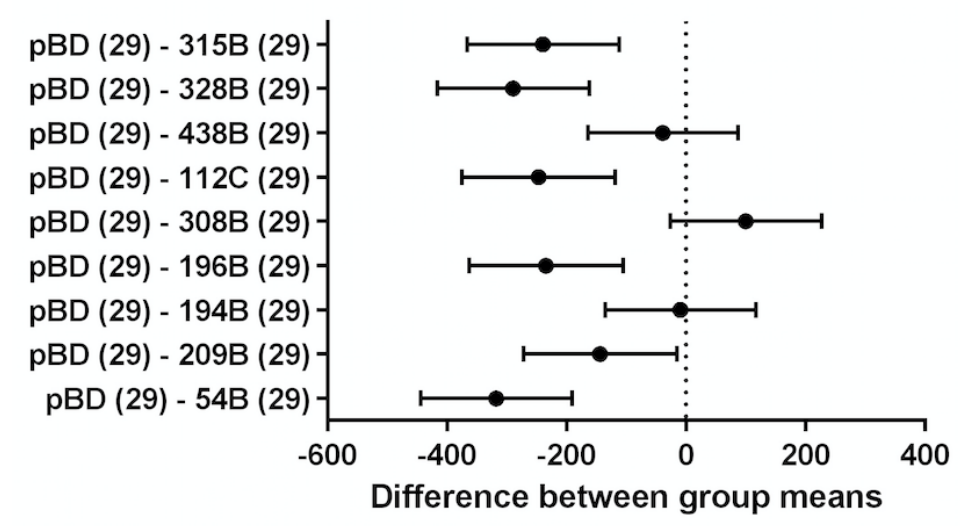

Figure 9. Confidence intervals from one-way ANOVA using Dunnet test

The data points represent the means of each genotype, and the bars represent the confidence level that the means of each genotype are statistically different from the control, pBD. The six genotypes, 54B, 209B, 196B, 112C, 328B, and 315B all show values with relatively high confidence that they are different from the control as they do not touch the dotted line.

\section{Discussion}

I hypothesized that activation of dopamine neurons in a receptor null background in specific dopamine neurons would result in more normal levels of sleep for those particular lines of flies. The experiments aimed to explore which, if any, of the pre-identified clusters of dopamine neurons controlled sleep in this way.

Previous research identified certain clusters of dopamine neurons in the mushroom body that, when chemo-genetically activated, would manifest by causing the fly to sleep less 
(Sitaraman et al. 2015). However, because this was a large screen that aimed to identify the clusters, there are still many unknowns regarding the specific roles and mechanisms of these clusters. Thus, our research aimed to explore these dopamine clusters more in depth so as to more fully understand the neural processes, with a specific focus on sleep.

In order to examine the effects of dopamine on sleep behavior, genetic techniques were used to insert a transgene into the fly's genome so that it would express these new instructions. The transgene, $\mathrm{dTrpA} 1$, is a chemosensitive cation channel that, when activated, causes hyperactivity of the dopamine neurons where it is expressed. This channel is activated by temperatures above $27^{\circ} \mathrm{C}$, so the flies were exposed to a $29^{\circ} \mathrm{C}$ environment to stimulate activation of dTrp to get an increase in dopamine release. However, the flies also were genetically manipulated so that they had non-functional dopamine receptors wherever there was activation of the dopamine neurons. This was done so that we could analyze the sleep patterns of the flies upon activation, as compared to non-activation, to see if this receptor null background manifested in any differences in sleep behavior. If a specific transgenic line of flies shows recovered sleep behavior, it could be evidence that it is crucial to controlling sleep. This is due to the fact that the non-functional dopamine receptors are mitigating any effects of the increased activity of the cation channel, and thus are giving us an indication that they are part of the pathway that controls sleep/wake behavior in the fruit fly.

The results from the experiment do appear to show certain clusters of dopamine neurons having more influence in controlling sleep behavior than others. In a sleep assay of ten separate Gal4 line crosses, the data show that during the first three days, when no activation of dTrpA1 occurred, there is very little difference in total sleep between the genotypes (Figure 7). This is logical and expected as the temperature is at $21^{\circ} \mathrm{C}$, and there should be no activation of the 
transgene, dTrpA1, which means the flies should have relatively normal levels of expression of dopamine. Thus, this is the data we use to establish their baseline behavior regarding sleeping and waking so that we can see if there are distinguishable differences when we activate our transgene dTrp. During activation day, the $29^{\circ} \mathrm{C}$ condition, we did see some interesting and even statistically significant genotypes. After running a one-way ANOVA followed by post-hoc correction, it was determined that six separate genotypes showed significant differences in their total sleep time. In the genotypes 54B, 209B, 196B, 112C, 328B, and 315B, the flies did not show the decreased sleep time that the other flies, including the control pBD showed. These six lines are sleeping amount the same amount as they were during the non-activation days at $21^{\circ} \mathrm{C}$. With activation of dTrp, there is more dopamine release, which should increase the activity of the flies and decrease their total sleep time. However, because we do not see this decrease in sleep in these six lines, we can tentatively conclude that the presence of the DopR2 null background is causing this recovery of sleep behavior. Because this receptor is not functional, it means that the increased dopamine levels cannot actually cause a behavioral change because no signals can be passed from one neuron to the next. In the genotypes that did show a decrease in sleep, we can hypothesize that those clusters of neurons are not centrally involved in controlling sleep, and it is the contribution of other neurons that cause their decreased sleep behavior. The genotypes that did show more regular total sleep time are likely crucial to controlling sleep because when we activate dTrp but essentially remove the ability for the communication to be transmitted, we are able to isolate the dopamine neurons that truly control sleep.

Overall, this data is important for identifying specific dopamine neuron clusters that control sleep in both fruit flies and eventually humans. Because of the complexity of the human brain, it is imperative that research starts in model organisms that are simpler and easier to 
understand. Therefore, research such as this is crucial in order to first learn how exactly dopamine neurons are controlling and regulating sleep behavior.

There are a couple important limitations that could potentially limit the strength of the conclusions. The first is that this data is mainly based on one experiment, and would therefore be greatly strengthened by completing more trials. If more experiments were to be completed, it would help to increase the strength of the data and be strong evidence that the six clusters are important for controlling sleep. More trails should not only be completed with the same genotypes, but also with other genotypes that may be implicated in controlling sleep behavior. Another limitation is that we cannot be completely sure that the effects we saw were singularly caused by the concurrent activation of dTrp with a receptor null background. There is a chance there is another mechanism occurring that may explain the recovered sleep behavior and we thus cannot be certain that the DopR2 null background is causing the significant effects in Figure 8.

\section{Conclusions and Future Research}

By utilizing genetic manipulation strategies and behavior experimental conditions, specific dopamine neurons were targeted in order to determine their role in controlling sleep behavior. In the collected data, it appears that despite activation of dopamine neurons through the use of dTrpA1, certain lines of flies are not having the sleep disturbances normally observed. Based on previous research and the results from this experiment, there is a reasonable amount of evidence to suggest that those lines of flies that are having more normal sleep behavior can recover that behavior because of the presence of non-functional dopamine receptors in particular dopamine neurons. It is likely that these specific dopamine neuron clusters are central to the neural control of sleep in the fly brain. 
The results show that there are six clusters that show statistically significant different levels of sleep, as compared to the control group: 54B, 209B, 196B, 112C, 328B, and 315B. This suggests that these six dopamine clusters are crucially involved with the neural control of sleep/wake behavior in the fruit fly.

However, this is only a small sample and more data is still being collected. If more data can be collected from the same lines that also shows similar trends, then there would be even more compelling evidence that the six statistically significant lines in the research so far are in fact important. Determining the neural mechanisms of the brain, even in the fruit fly, is difficult, and thus it is important that this research and similar research continues to investigate these mechanisms. Determining the role and mechanisms of dopamine neurons controlling sleep will not only help the neuroscience community to better understand the brain of Drosophila melanogaster, but eventually the human brain as well.

\section{Dopamine Sleep Disorders}

In order to give some context to the implications of the research, this section will cover a few different sleep disorders seen in humans that have a strong dopamine component. The first is Parkinson's disease. Although not the main component of the disease, many of those suffering from Parkinson's disease (PD) experience sleep dysfunction due to the abnormal dopamine levels that are present in PD. The most common symptoms of sleep disturbance are an inability to sleep through the night and difficulty falling back asleep once awakened. This is often referred to as maintenance insomnia because of the persistent nature and severity of the sleep loss. People with Parkinson's report daytime sleepiness, nightmares, vivid dreams, and nighttime vocalizations ("Parkinson's Disease” 2017). There is evidence to show that dopamine plays a 
crucial role in maintaining wakefulness, and in Parkinson's patients who often have too high or too low levels of dopamine, it is common to see these sleep dysfunctions (Mehta 2008). PD is characterized by low dopamine in the brain, thus patients can get sleep disturbances from the low levels of dopamine, as well as problems when their dopamine-increasing medications raise their levels of dopamine too much.

Another sleep disorder is that found in people with schizophrenia. Schizophrenia is generally known to have a strong biochemical component of higher dopamine levels as compared with those who are not diagnosed with the disorder (Dzirasa 2006). As seen in Parkinson's, when dopamine levels are abnormal, there is often sleep dysfunction, and schizophrenia is no different. People with schizophrenia often report getting less sleep and report "dreaming while awake" (Gottesmann 2008). This is a relatively newly described symptom that basically involves the person being unable to distinguish reality from dreams, and often occurs just after waking. Just as seen in PD, when dopamine medications are administered, there can be detrimental sleep side effects if the medications do not properly control dopamine levels.

A final sleep disorder is restless leg syndrome. Restless leg syndrome, also called WillisEkbom Disease, is characterized by unpleasant or uncomfortable sensations in the legs with a subsequent urge to move them to relieve the discomfort ("National Institute of Neurological Disorders and Stroke” 2017). This often occurs during sleep, which categorizes it as a sleep disorder. There is also evidence of a strong dopamine component and its relation to how dopamine control both sleep and muscle movements. Recent research has shown that there could be a possible decrease in dopamine receptors, with a simultaneous increase in production and release of dopamine (Salas 2016). This seems to be a compensation effect in order to make up 
for the reduction in receptors, but it over-compensates and results in too much dopamine, which in turn causes the effects seen in RLS.

Overall, research on specific dopamine neurons that control sleep is important because it could potentially lead to treatments that would be able to directly target the dopamine neurons that are causing sleep dysfunction. It is valuable to learn about the structures and mechanisms of the brain for academic purposes as well as clinical ones, and this type of research could be important in treating those who suffer from sleep disorders. If we can identify the specific neuron clusters that control sleep in the human brain, there is the possibility of developing medications that could act locally to increase or decrease dopamine, instead of simply generally increasing dopamine all throughout the brain. 


\section{References}

Dzirasa, K., S. Ribeiro, R. Costa, L. M. Santos, S. C. Lin, A. Grosmark, T. D. Sotnikova, R. R. Gainetdinov, M. G. Caron, and M. A. Nicolelis. "Dopaminergic Control of Sleep-wake States." The Journal of Neuroscience : The Official Journal of the Society for Neuroscience. U.S. National Library of Medicine, 11 Oct. 2006. Web. 13 Apr. 2017.

Gottesmann, Claude. "The Neurochemistry of Waking and Sleeping Mental Activity:

The Disinhibition-dopamine Hypothesis." Psychiatry and Clinical Neurosciences. Blackwell Science Pty, 04 Aug. 2008. Web. 1 May 2017.

Heisenberg, Martin. "What Do the Mushroom Bodies Do for the Insect Brain? An Introduction." Learning \& Memory. Cold Spring Harbor Laboratory Press, 5 May 1998. Web. 28 Mar. 2017.

Koenig, Sebastian, Reinhard Wolf, and Martin Heisenberg. "Visual Attention in FliesDopamine in the Mushroom Bodies Mediates the After-Effect of Cueing." PLOS ONE. Public Library of Science, 29 Aug. 2016. Web. 3 May 2017.

Mehta, S. H., J. C. Morgan, and K. D. Sethi. "Sleep Disorders Associated with Parkinson's Disease: Role of Dopamine, Epidemiology, and Clinical Scales of Assessment." CNS Spectrums. U.S. National Library of Medicine, 13 Mar. 2008. Web. 15 Apr. 2017.

National Institute of Neurological Disorders and Stroke. National Institutes of Health. U.S. Department of Health and Human Services, 2017. Web. 25 Apr. 2017.

Oishi, Y., and M. Lazarus. "The Control of Sleep and Wakefulness by Mesolimbic Dopamine Systems." Neuroscience Research. U.S. National Library of Medicine, 20 Apr. 2017. Web. 1 May 2017.

"Parkinson's Disease Clinic and Research Center." Sleep and Parkinson's Disease | Parkinson's 
Disease Clinic and Research Center. N.p., n.d. Web. 2 May 2017.

Potdar, S., and V. Sheeba. "Lessons from Sleeping Flies: Insights from Drosophila Melanogaster on the Neuronal Circuitry and Importance of Sleep." Journal of Neurogenetics. U.S. National Library of Medicine, 27 June 2013. Web. 26 Apr. 2017.

Salas, Rachel E., Charlene E. Gamaldo, and Richard P. Allen. "Update in Restless Legs Syndrome." Current Opinion in Neurology. U.S. National Library of Medicine, 28 June 2016. Web. 13 May 2017.

Sitaraman, Divya, Yoshinori Aso, Gerald M. Rubin, and Michael N. Nitabach. "Control of Sleep by Dopaminergic Inputs to the Drosophila Mushroom Body." Frontiers. Frontiers, 23 Oct. 2015. Web. 1 May 2017.

Tomita, J., G. Ban, and K. Kume. "Genes and Neural Circuits for Sleep of the Fruit Fly." Neuroscience Research. U.S. National Library of Medicine, 21 Apr. 2017. Web. 26 Apr. 2017.

Wisor, Jonathan P., Seiji Nishino, Ichiro Sora, George H. Uhl, Emmanuel Mignot, and Dale M. Edgar. "Dopaminergic Role in Stimulant-Induced Wakefulness." Journal of Neuroscience. Society for Neuroscience, 01 Mar. 2001. Web. 1 May 2017.

Yates, N. J. "Schizophrenia: The Role of Sleep and Circadian Rhythms in Regulating Dopamine and Psychosis." Reviews in the Neurosciences. U.S. National Library of Medicine, 01 Oct. 2016. Web. 13 Mar. 2017. 